\title{
Borehole IP anomaly characteristics analysis of the single well and the double wells working way
}

\author{
Tao Liu ${ }^{1}$, Jiao Luo $^{1} \&$ Xiangwen Zhou ${ }^{2}$ \\ ${ }^{1}$ College of Geology Engineering and Geomatics, Chang'an University, Xi'an, 710054, China \\ ${ }^{2}$ Transportation Research Institute, Shanxi, 030006, China
}

Keywords: Borehole IP, well, apparent resistivity, apparent polarizability.

\begin{abstract}
Borehole IP method is an important branch of the well electrical prospecting method. It is a flexible way of working, which is widely used in the resource investigation and engineering geological survey, or other fields, and achieved good results. This paper introduces the way well induced polarization method working in the field which is based on the single well and double wells. Last but not the least, the Author calculated the apparent resistivity and apparent polarizability to the vertical plate and mapped the apparent resistivity and apparent polarizability profile under the way of single well and double Wells, analysing the relationship between the anomalies and blind ore body. After analysing the anomaly characteristics of the vertical plate shaped body in single well and double wells, we can draw the following conclusions: the apparent polarizability anomaly response is generally better than the apparent resistivity abnormal reaction; depending on the apparent polarizability profile, we can find that apparent polarizability is almost not affected by the surface and topography, and the apparent resistivity can be influenced by the surface topography some extent. So the apparent polarizability can be used as main parameters to the single well and the double wells in the borehole IP and the apparent can be used as a secondary parameter.
\end{abstract}

\section{Introduction}

With the rapid development of human civilization, the earth resources and energy consumption is becoming more and more serious, especially in our country, so the exploration and exploitation of energy and mineral resources has become one of the primary task of economic construction of our country. China is not only the amount of resources per capita less, but also conducive to the development of shallow deposits have been found in mined out, many of the production of Mines due to resource depletion and bankrupt.

Borehole induced polarization is based on the difference of the rocks and minerals in induced polarization effects. Through observation and research, we can explore the underground geological conditions. As a expand application to the common ground induced polarization in the borehole, borehole IP method can be used to evaluate the ground induced polarization anomalies and increased exploration depth. Because of the in-hole powering or measuring ,detection equipment's is closer to underground targets, so those affects from the ground noise and surface covers get smaller. It can increase the induced polarization response and the exploration depth. Therefore, borehole induced polarization method is getting more attention recent years and its development and application level continues to increase. It is unique in many aspects. For example, the field source and receive equipment are into the deep underground wells and the different detection instruments approach or through the ore body from different directions, thereby it can increase the degree of abnormal display and effectively improve the ability to find deep mine. Also, it avoids the absorption of the ground geophysical mulch to the field source.

\section{Field work methods}

This paper selected two observation modes, including single well mode and double wells mode. 


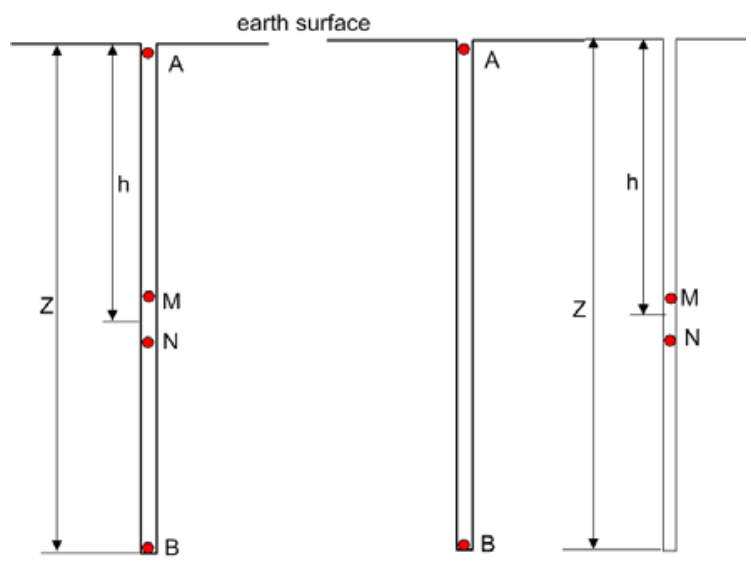

(a)

(b)

\section{Single well gradient IP}

Single well gradient array is placeing pole A to the wellhead or near the position and pole B is arranged in the bottom (Fig.1a). The $\mathrm{M}$ and $\mathrm{N}$ electrodes ( $\mathrm{M}$ over $\mathrm{N}$ ) are at Interval of 5 10 meters, measuring in the range of $\mathrm{A}$ and $\mathrm{B}$ along the well. The supplied-current intensity is selected within the range of $1 \sim 3 \mathrm{~A}$. The main parameter of this approach is apparent polarization, which is combined with the apparent resistivity.

\section{Double Wells gradient IP}

Similarly to the single well gradient array, the double wells gradient array is more complex, the array is placeing pole $A$ to the wellhead or near the wellhead and pole $B$ is arranged in the bottom (Fig.1b). $\mathrm{M}$ and $\mathrm{N}$ electrodes move in another well with measurement. The parameter selections may refer to the single well. The normal field of this arrangement is relatively stable and does not appear potential difference to zero value point. We can choose secondary field and parameters such as apparent resistivity or apparent polarization flexible in order to obtain the most obvious IP anomalies.

Borehole IP method has the advantage that it can avoid the influence of electrical inhomogeneity caused by uneven terrain and covering, especially the shielding effect of low resistivity layers. Thereby it significantly increase the exploration depth and can choose flexible way to observe.

\section{The vertical plate shaped body borehole IP forward calculation}

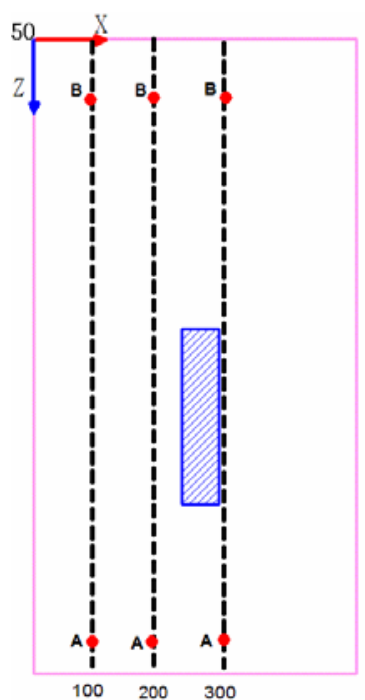

(a)

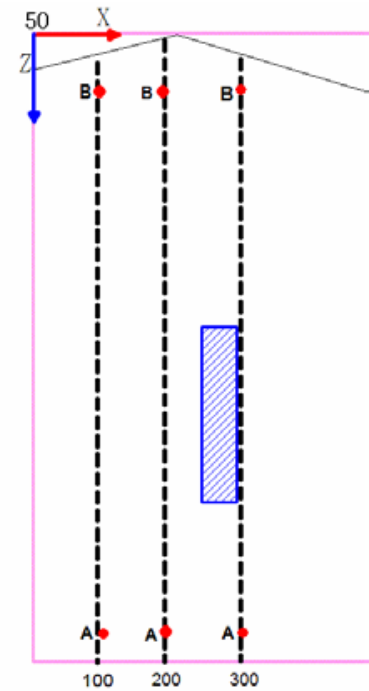

(b)

Fig. 2: schematic diagram to terrain model and level terrain model

The vertical plate shaped body model's size is $50 \mathrm{~m} * 300 \mathrm{~m} * 250 \mathrm{~m}$. The detailed parameters as follows, $\mathrm{X}$ direction stretches $250 \mathrm{~m}$ to $300 \mathrm{~m}$, Y direction stretches $-100 \mathrm{~m}$ to $200 \mathrm{~m}$, Z direction 
stretches the depth of $-400 \mathrm{~m}$ to $-650 \mathrm{~m}$, the resistivity is $300 \mathrm{ohm}$. $\mathrm{m}$, the polarization rate is $20 \%$; the background resistivity is $3000 \mathrm{ohm}$. $\mathrm{m}$, the polarization rate is $3 \%$, the supply current is $1 \mathrm{~A}$. The schematic diagram of the model is shown in Fig. 2, the black dashed line in the diagram is well position, the blue rectangle for the anomalous body. The main view take $y=50 \mathrm{~m}$ 's main section and the well positions as shown in the figure are $(100,50),(200,50)$ and $(300,50)$. When it supplies power in the well located in $(200,50)$, we can measure in the well $(100,50),(200,50)$ and $(300,50)$. According to the preset model and the data acquisition mode, we can calculate the apparent resistivity apparent polarizability of the wells under three kinds of situations, and mapped the apparent resistivity and apparent polarizability profile pictures in Fig. 3, Fig. 4 and Fig. 5.
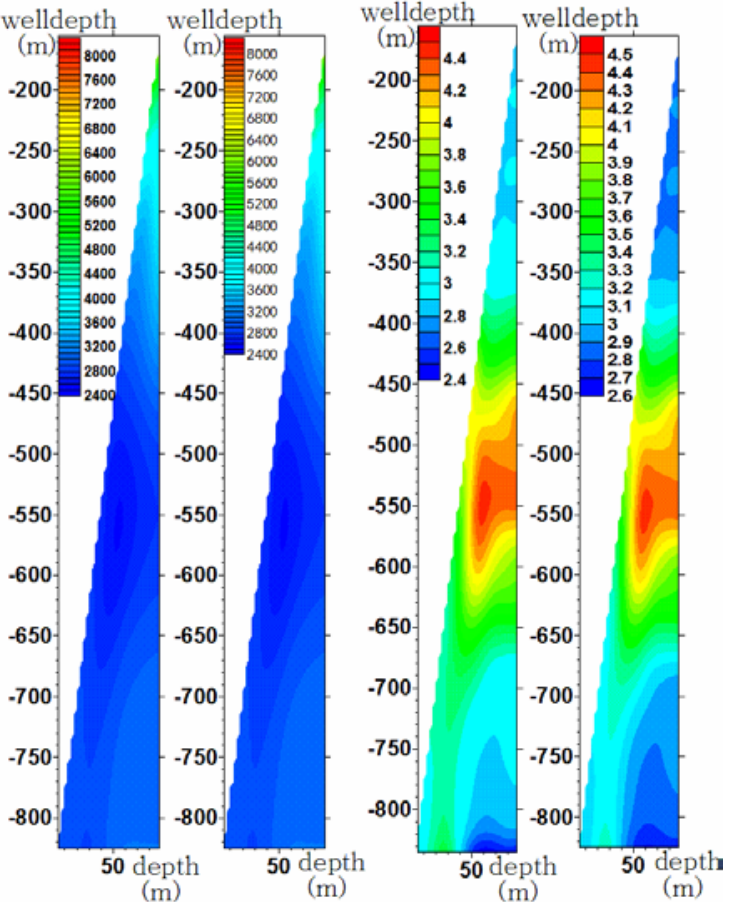

Fig. 3 the apparent resistivity profile and the apparent polarizability profile in $(100,50)$ well

There are four profile picture in Fig. 3. When it supplies power in the well located in $(200,50)$, we measure the anomalies in the well $(100,50)$. Every two picture is a group, the first picture of the first group is with terrain ground well apparent resistivity profile and the second is level terrain ground well apparent resistivity profile. The second group is the apparent polarizability profile to the $(100,50)$ well, the first picture of the second group is with terrain ground well apparent polarizability profile and the second is level terrain ground well apparent polarizability profile

From Fig.3 we can draw some conclusions. On the whole, as can be seen from the two cases to the (100,50) well , because abnormal body is far from the well, so the apparent resistivity anomaly is not very obvious and abnormal value is relatively small. In the case with the terrain, the high resistivity anomaly to the upper part of the profile can reflect the influence of the terrain but the level terrain without this high resistivity reaction. Although he anomaly amplitude is small, but it can basically reflect anomalies of the upper and lower range.

On the other hand, the apparent polarizability profile can reflect the high polarization characteristics of the abnormal body. The more the measuring well close to the abnormal body, the more apparent polarizability anomaly response is obvious. Specifically to see, as can be seen from the two cases to the $(100,50)$ well, because abnormal body is far from the well, though the apparent polarizability anomaly is very obvious but the abnormal value is relatively small. The two pictures in the second group are very similar in form, indicating the weak impact of the terrain and the small change value. 

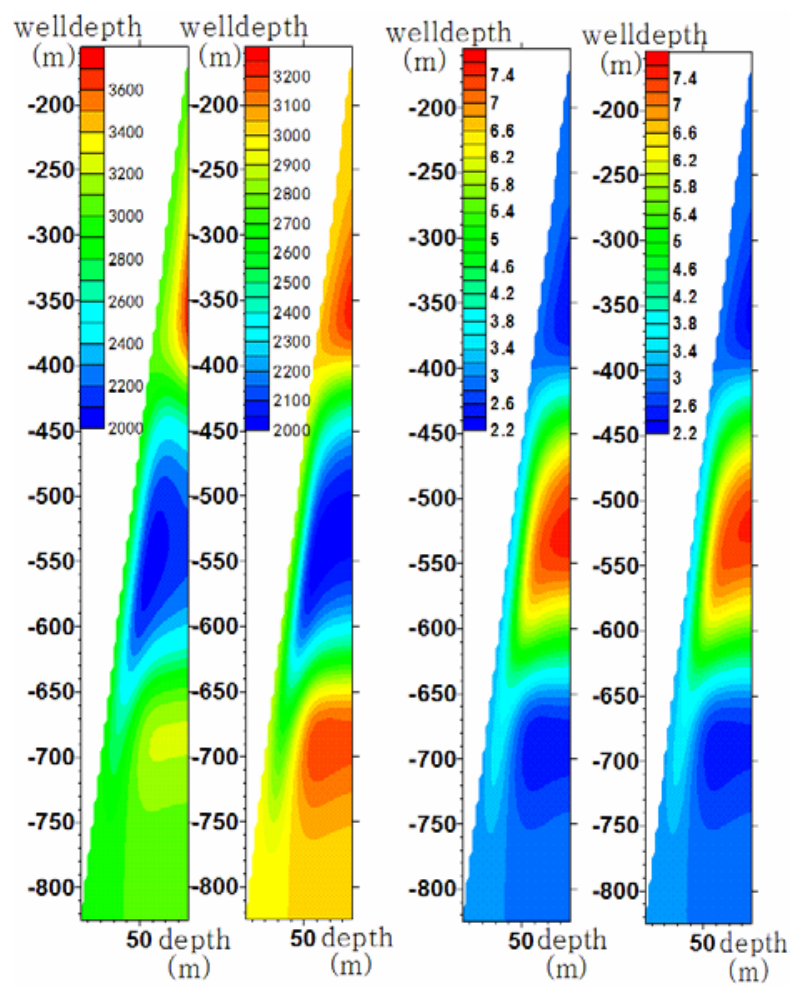

Fig. 4 the apparent resistivity profile and the apparent polarizability profile in $(200,50)$ well

There are four profile picture in Fig. 4. When it supplies power in the well located in $(200,50)$, we also measure the anomalies in the well $(200,50)$. Every two picture is a group, the first picture of the first group is with terrain ground well apparent resistivity profile and the second is level terrain ground well apparent resistivity profile. The second group is also the apparent polarizability profile to the $(200,50)$ well, the first picture of the second group is with terrain ground well apparent polarizability profile and the second is level terrain ground well apparent polarizability profile.

From Fig.4 we can draw some similar conclusions. On the whole, as can be seen from the two cases to the (200,50 ) well , compared to the $(100,50)$ well, because abnormal body is far from the well, so the apparent resistivity anomaly is not very obvious and abnormal value is relatively small. In the case with the terrain, the high resistivity anomaly to the upper part of the profile can reflect the influence of the terrain and the level terrain also can reflect this high resistivity reaction obviously. Because the anomaly amplitude increased obviously, so it can reflect anomalies of the upper and lower range more clearly.

From the second group of the Fig.4, we can also get some conclusions. The more the measuring well close to the abnormal body, the more apparent polarizability anomaly response is obvious. Specifically to see, as can be seen from the two cases to the $(200,50)$ well, because abnormal body is closer to the $(200,50)$ well than the $(100,50)$ well, so the apparent polarizability anomaly is more obvious and the abnormal value is bigger, indicating the weak impact of the terrain and the relatively big change value.

Depending on the apparent polarization profile shown in Fig. 5, we can analyze the characteristics of the apparent polarizability. Fig. 5 has the same sequence with Fig. 3 and Fig. 4 on the picture sort and details are as above. Power supply well is also located in the $(200,50)$ well, we also measure the anomalies in the well $(300,50)$ this time.

From Fig. 5 we can draw some conclusions, on the whole, as can be seen from the two cases in the $(300,50)$ well, compared to the results measured in the $(100,50)$ well and the $(200,50)$ well, because abnormal body is very close to the $(300,50)$ well, so the apparent resistivity anomaly is very obvious and abnormal value is even bigger. In the case with the terrain, the high resistivity anomaly to the upper part of the profile can reflect the influence of the terrain and the level terrain also can reflect this high resistivity reaction obviously. But the anomaly amplitude increased 
obviously, so it can reflect anomalies of the upper and lower range more clearly. What' more, because abnormal body is very close to the $(300,50)$ well, the influence of terrain has been suppressed, and it can well reflect the scope of the abnormal body and assignment.

On the whole, the apparent polarizability profile can reflect the high polarization characteristics of the abnormal body. The more the measuring well close to the abnormal body, the more apparent polarizability anomaly response is obvious. As can be seen from the two cases to the (300,50) well , because abnormal body is very close to the well, so the apparent polarizability anomaly is very obvious and the abnormal value is even bigger than the measured results in the $(200,50)$, with the little impact of the terrain and the big change value. The apparent polarizability of the ( 300,50$)$ well is similar to the case of the (200,50) well , but the $(300,50)$ well is closer to the abnormal body, so the maximum value gets bigger and the maximum value is very close to the true polarizability of the abnormal body .
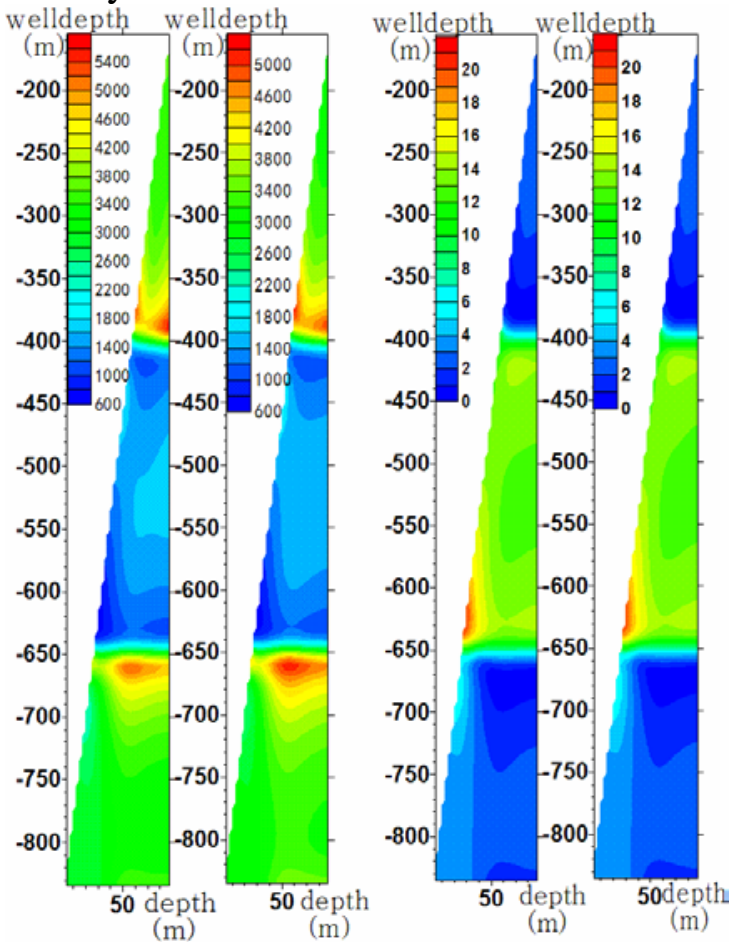

Fig. 5 the apparent resistivity profile and the apparent polarizability profile in $(300,50)$ well

\section{Conclusions}

For the two cases with terrain and level terrain, borehole IP apparent resistivity profile and apparent polarizability profile indicate that apparent polarizability anomaly is almost not affected by these two cases but apparent resistivity anomaly gets certain influence. Comparative analysis shows that, the more the measuring well close to the abnormal body, the better is the detection effect to resistivity anomaly. The abnormal reaction of apparent polarizability is generally better than the abnormal response of the apparent resistivity. Through supplying power to all wells and measuring in all wells (including single well and double wells), firstly, we can obtain the apparent resistivity and apparent polarizability under the different circumstance; secondly, we can get the location of the abnormal body that relative to the well; thirdly, we can acquire the direction of abnormal body and the range of the abnormal value.

\section{Acknowledgements}

The research work was supported by National Natural Science Foundation of China under Grant No. 61065009 and Natural Science Foundation of Qinghai Provincial under Grant No. 2011-z-756. 


\section{References}

[1] Mitsuhata.Y. 2D electromagnetic modeling by finite element method with a dipole source and topography [J]. Geophysics, 2000, 65(2); 465-475

[2] A.F.Jepsen. Resistivity and induced polarization modeling [D].Berkele University of California, 1969

[3] K.Spitzer \& B. Wurmstich. Speed and accuracy in three-dimensional resistivity modeling [J].

Geophysics, 2001, 66 (5):598-613

[4] G.W. Hohmann. Three-dimensional induced polarization and electromagnetic modeling [J]. Geophysics, 1975, 40(2):309-324

[5] Gary Barnes. Reconstructing the gravity gradient anomaly field from surveys with wide line spacing using equivalent source processing: an error analysis [J]. Geophysical Prospecting, 2014, 62(3).

[6] T. Al-Wahaibi, Y. Al-Wahaibi, A. Al-Ajmi et al.. Experimental investigation on flow patterns and pressure gradient through two pipe diameters in horizontal oil-water flows [J]. Journal of Petroleum Science and Engineering, 2014, 122.

[7] G Ramadass, S M Varaprasada Rao, N L Mohan. Base metal exploration by gravity surveys in a part of the Cuddapah basin (India) — A case study [J]. Proceedings of the Indian Academy of Sciences - Earth and Planetary Sciences, 1986, 95(1).

[8] S. V. Lavrikov, A. F. Revuzhenko. One experimental rock model [J]. Soviet Mining Science, 1992, 27(4).

[9] Francesca Casini, Cristina Jommi, Sarah Springman. A laboratory investigation on an undisturbed silty sand from a slope prone to landsliding. Granular Matter, 2010, 12(3). 